\title{
EFEITO DOS PROCEDIMENTOS \\ DE ACABAMENTO E POLIMENTO \\ NA RUGOSIDADE SUPERFICIAL DA \\ CERÂMICA NORITAKE ${ }^{\circledR}$
}

\author{
Mario Cezar Oliveira*, Alex Correia Vieira*, Adriana Castro Andrade*, Indira Almeida Oliveira** \\ Autor correspondente: Mario Cezar Oliveira - mcezar11@gmail.com \\ * Professor Adjunto da Área de Prótese Dentária da Universidade Estadual de Feira de Santana \\ ** Aluna do curso de graduação em Odontologia da Universidade Estadual do Sudoeste da Bahia
}

\begin{abstract}
Resumo
O objetivo desse estudo foi avaliar a rugosidade superficial da cerâmica Noritake $₫$ após diferentes métodos de acabamento e polimento. Foram confeccionados 40 corpos de prova da cerâmica Nori-

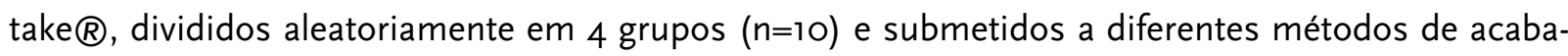
mento e polimento: Grupo 1 (controle) - aplicação do glaze; Grupo 2 - borrachas abrasivas Astropol (Ivoclar Vivadent), disco de feltro e pasta diamantada; Grupo 3 - borrachas abrasivas (Edenta), disco de feltro e pasta diamantada; Grupo 4- discos Sof-Lex (3M-ESPE), disco de feltro e pasta diamantada. Após a realização destes procedimentos, todos os corpos de prova foram submetidos a análise quantitativa da rugosidade superficial em rugosímetro Mitutoyo SI 301 . O grupo controle $\left(G_{1}\right)$ apresentou a menor média de rugosidade superficial $(0.68 \mu \mathrm{m})$, sendo estatisticamente diferente dos demais grupos $(\mathrm{p}<0.001)$, que tiveram valores superiores de rugosidade de $1.11 \mu \mathrm{m}\left(\mathrm{G}_{2}\right), 1.12 \mu \mathrm{m}\left(\mathrm{G}_{3}\right)$ e 1.14 $\mu \mathrm{m}\left(\mathrm{G}_{4}\right)$. Porém, estes grupos não apresentaram diferenças estatisticamente significante entre si ( $p>0.05)$. Os diferentes métodos de acabamento e polimento não foram capazes de promover uma superfície tão lisa quanto à aplicação do glaze na cerâmica testada.
\end{abstract}

Palavras-chave: Cerâmica; Ajuste Oclusal; Polimento Dentário. 


\title{
THE EFFECT OF FINISHING AND POLISHING PROCEDURES ON THE SURFACE ROUGHNESS OF NORITAKE ${ }^{\circledR}$ CERAMIC
}

\begin{abstract}
The aim of this study was to evaluate the surface roughness of Noritake $\mathbb{R}$ ceramic after different methods of

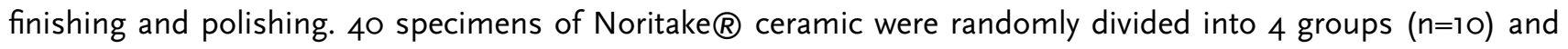
subjected to different methods of finishing and polishing: Group 1 (control) - glaze; Group 2 - abrasive rubbers Astropol (Ivoclar Vivadent), felt disc and diamond paste; Group 3 - abrasive rubbers (Edenta), felt disc and diamond paste; Group 4 - Sof-Lex discs (3M ESPE), felt disc and diamond paste. Subsequently, the surface roughness was measured with rugosimeter (Mitutoyo S/ 301). The control group (G1) had the lowest surface roughness $(0.68 \mu \mathrm{m})$ that was different statistically to the other groups $(p<0.001), G_{2}(1.11 \mu \mathrm{m}), G_{3}(1.12 \mu \mathrm{m})$ e $G_{4}(1.14 \mu \mathrm{m})$. Comparing the values of $R a$ from $G_{2}, G_{3}$ and $G_{4}$ with each other, statistically significant differences were not found ( $p>0.05$ ). The different methods of finishing and polishing were not able to promote a surface as smooth as the application of glaze in the ceramics tested.
\end{abstract}

Keywords: Ceramics; Occlusal Adjustment; Dental polishing.

\section{INTRODUÇÃO}

Atualmente, existe uma busca constante pela beleza, tecnologia e perfeição. Assim, o desenvolvimento de materiais que consigam suprir as necessidades estéticas está cada vez mais acelerado. $\mathrm{E}$ neste contexto, pacientes que procuram recursos para o sorriso perfeito, têm sido uma constante na rotina dos dentistas. ${ }^{(1)}$

Desde sua introdução na odontologia, as cerâmicas têm merecido atenção e destaque, e a principal razão para isso é que, até então, nenhum outro material consegue reproduzir a beleza e a naturalidade dos dentes. Deste modo, o uso das cerâmicas passou a ser amplamente difundido na sociedade com o intuito de atender às necessidades estéticas e funcionais. ${ }^{(2)}$

Dentre as propriedades físicas e estéticas encontradas nas cerâmicas odontológicas, ${ }^{(3,4)}$ pode-se destacar como vantagens a sua estabilidade de cor, reprodução de textura superficial, opalescên- cia e translucidez, (5) baixa condutibilidade térmica e elétrica, durabilidade, radiopacidade, resistência à degradação e desgaste no meio oral, além da biocompatibilidade tecidual. ${ }^{(5-7)}$

Apesar de todas estas vantagens, as restaurações realizadas com este material, após a sua cimentação na estrutura dentária, necessitam passar por ajustes intraorais com pontas diamantadas, para que haja uma perfeita oclusão com o dente antagonista e para corrigir alterações no contorno e forma das restaurações, promovendo uma meIhor estética das mesmas. ${ }^{(2,7,8)}$

Estes ajustes, por sua vez, poderão acarretar em superfícies altamente rugosas que comprometerão a estética e a saúde gengival, causando desgaste de dentes e/ou restaurações antagonistas, acúmulo de biofilme e consequentemente, inflamação dos tecidos moles. ${ }^{(2,3,8)}$ Com o intuito de minimizar tais inconvenientes, preconiza-se a utilização 
de técnicas de acabamento e polimento intraoral como o uso de pedras montadas, discos de óxido de alumínio e borrachas abrasivas, com o objetivo de obter superfícies lisas o bastante para promover a biocompatibilidade das restaurações com os tecidos adjacentes. ${ }^{(6,9)}$

Um novo processo de glazeamento era adotado como o melhor procedimento a ser realizado após os ajustes com pontas diamantadas, entretanto, passou a ser utilizado com maior restrição, já que aumentava o valor das restaurações, refletindo mais luz do que os dentes naturais, proporcionado um aspecto artificial. ${ }^{(5,8,10)} \mathrm{Em}$ contrapartida, os polimentos manuais passaram a ser mais utilizados, pois removem o brilho excessivo gerado pelo processo de glazeamento, proporcionando às restaurações um aspecto natural, além de reduzir o tempo clínico de trabalho, já que a restauração não necessita retornar ao laboratório para a reaplicação do glaze. Apesar destes achados, existem controvérsias quanto à eficácia destes polimentos na obtenção de superfícies lisas e biocompatíveis com os tecidos adjacentes. ${ }^{(8)}$

Dentro deste contexto, a avaliação de diferentes métodos de acabamento e polimento de restaurações em cerâmica, surge com o intuito de desvendar a eficácia na obtenção de restaurações estéticas e biocompatíveis, ao modo que servirá de orientação para profissionais que buscam a melhor técnica para unir estética, função e saúde dos tecidos dentais e periodontais. Com este propósito, o objetivo desse estudo foi avaliar a rugosidade super-

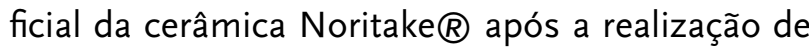
diferentes métodos de acabamento e polimento.

\section{MATERIAL E MÉTODOS}

\section{CONFECÇÃO DOS CORPOS DE PROVA}

Foram confeccionados 40 corpos de prova cerâmicos utilizando-se uma matriz retangular de alumínio com $10 \mathrm{~cm}$ de comprimento e $3 \mathrm{~cm}$ de largura, possuindo 7 perfurações com $0,5 \mathrm{~cm}$ de diâmetro interno por $0,2 \mathrm{~cm}$ de espessura, determinando assim as dimensões dos corpos de prova. A cerâmica utilizada foi a feldspática Noritake $®$ (Nagoya, Aichi, Noritake Dental Supply Co, Japão), na cor $A_{3} B$, em laboratório de prótese dental e as amostras foram confeccionadas por um mesmo operador.

Para todos os corpos de prova, o pó cerâmico foi incorporado à água destilada e manipulado até se atingir a consistência de massa, de acordo com as recomendações do fabricante. Após a remoção dos excessos de água com papel absorvente, a cerâmica foi levada às perfurações da matriz até o seu completo preenchimento. Os corpos de prova foram removidos das perfurações, após 1 minuto, por compressão digital suave, para que não ocorresse fratura, e em seguida, foram levados ao forno Vacumat 4OT (VITA Zahnfabrik, Alemanha) para queima.

Foram confeccionados apenas 05 corpos de prova por período de queima. Estes foram submetidos a uma queima com temperatura inicial de $550^{\circ} \mathrm{C} \mathrm{e}$ temperatura final de $940^{\circ} \mathrm{C}$, com vácuo total. Depois de resfriadas, as superfícies foram asperizadas com jatos de óxido de alumínio de $50 \mu$ e aplicado o glaze de acordo com a recomendação do fabricante.

\section{DESGASTE SIMULANDO}

\section{AJUSTE OCLUSAL}

Posteriormente, todos os corpos de prova, com exceção dos 10 que formam o Grupo 1 (controle), permanecendo com sua superfície glazeada, foram fixados em uma placa de vidro com cera pegajosa (ASFER - Indústria Química LTDA, SP, Brasil), para realização do desgaste superficial com ponta diamantada $2135 \mathrm{~F}$ (KG Sorensen, Baureri, SP, Brasil) adaptada em peça de mão de alta rotação (KaVo do Brasil Ind. Com. LTDA, Joinville, SC, Brasil) para simular o ajuste oclusal. O desgaste foi realizado por um mesmo operador, utilizando movimentos suaves, durante 10 segundos, com refrigeração ar/água. 
Uma mesma ponta diamantada foi usada para 05 corpos e em seguida descartada.

Após a realização dos desgastes as amostras foram distribuídas aleatoriamente em 03 grupos $(n=10)$, que receberam diferentes métodos de acabamento e polimento.

\section{POLIMENTO DOS CORPOS DE PROVA}

O grupo controle $\left(G_{1}\right)$ contém 10 corpos de prova glazeados, os quais não sofreram qualquer desgaste ou polimento.

O grupo $\mathrm{G}_{2}$ foi submetido ao polimento com borrachas abrasivas da marca Ivoclar Vivadent (Astropol - Barueri, São Paulo, Ivoclar Vivadent, Brasil). Cada corpo de prova foi polido com três borrachas de diferentes granulações, uma de granulação mais grossa, mais abrasiva (Astropol F - Cinza) para pré-polimento, em seguida, uma de granulação intermediária (Astropol P - Verde) para polimento e por último, uma de granulação mais fina (Astropol HP - Rosa) para polimento de alto brilho. Todas as borrachas foram presas a uma peça de mão de baixa rotação (KaVo do Brasil Ind. Com. LTDA, Joinville, SC, Brasil), utilizada em movimentos leves de vaivém durante 15 segundos. Em seguida, os corpos de prova foram polidos com disco de feltro Diamond (FGM, Joinville, SC, Brasil) e pasta diamantada (Diamond Excel, FGM, Joinville, SC, Brasil), montados com mandril em contra-ângulo e utilizados da mesma forma e durante o mesmo tempo das borrachas abrasivas.

O grupo $\mathrm{G}_{3}$ recebeu polimento com borrachas abrasivas da marca Edenta (Edenta Ag Dental Produkt, Suiça). Cada corpo de prova foi polido com três borrachas de diferentes granulações, a primeira de granulação mais grossa (EXA CERAPOL branco/cinza) para um pré-polimento, em seguida, uma de granulação intermediária (EXA CERAPOL ROSA - rosa) para o polimento propriamente dito e por último, uma de granulação mais fina (CERAPOL SUPER - cinza) para polimento de alto brilho. Todas as borrachas foram presas a uma peça de mão de baixa rotação (KaVo do Brasil Ind. Com.
LTDA, Joinville, SC, Brasil), utilizada em movimentos leves de vaivém durante 15 segundos. Em seguida os corpos de prova foram polidos com disco de feltro Diamond (FGM, Joinville, SC, Brasil) e pasta diamantada (Diamond Excel, FGM, Joinville, SC, Brasil), montados com mandril em contra-ângulo e utilizados também em movimentos leves de vaivém durante 15 segundos.

O grupo $\mathrm{G}_{4}$ recebeu polimento com discos de óxido de alumínio (Discos Sof-Lex 3M ESPE, SP, Brasil). Cada elemento foi polido com três discos de granulações diferentes. O primeiro de granulação mais grossa, em seguida, um de granulação intermediária e posteriormente, um de granulação mais fina, presos a uma peça de mão de baixa rotação (KaVo do Brasil Ind. Com. LTDA, Joinville, SC, Brasil). Em seguida os corpos de prova foram polidos com disco de feltro Diamond (FGM, Joinville, SC, Brasil) e pasta diamantada (Diamond Excel, FGM, Joinville, SC, Brasil), montados com mandril em contra-ângulo e utilizados com o mesmo tempo e movimento dos grupos anteriormente citados.

\section{ANÁLISE DA RUGOSIDADE SUPERFICIAL}

Após os procedimentos de acabamento e polimento, os corpos de prova foram lavados em ultrassom, imersos em água destilada, durante 10 minutos e em seguida secos com papel absorvente.

Cada corpo de prova foi então fixado com uma fita dupla face em uma placa de vidro e submetido à leitura em rugosímetro Surftest SI-301 (Mitutoyo, Jp), para determinação da rugosidade superficial. A leitura considerada foi a média aritmética $(R a)$ entre os picos e vales percorridos pela ponta ativa do aparelho, onde o percurso de medição foi de 4,0 $\mathrm{mm}$. Foram realizadas três leituras na superfície de cada corpo de prova: uma no sentido horizontal, outra perpendicular a primeira e uma no sentido oblíquo. As médias dos valores obtidos foram anotadas, tabuladas e submetidas à análise estatística através da análise de variância e teste de Tukey, nível de significância de $5 \%$ para comparações múltiplas. 


\section{RESULTADOS}

A tabela 1 apresenta os valores da rugosidade superficial média ( $\mathrm{Ra}$ ) e desvio-padrão (dp) dos grupos testados. De acordo com os resultados encontrados, a rugosidade superficial foi influenciada significantemente pelo tipo de acabamento e polimento realizado na superfície das amostras de ce- râmica $(\mathrm{p}<0.001)$. O grupo controle $(\mathrm{G} 1)$ apresentou a menor média de rugosidade superficial ( 0.68 $\mu \mathrm{m})$, sendo estatisticamente diferente dos demais grupos $(p<0.001)$, que tiveram valores superiores de rugosidade de $1.11 \mu \mathrm{m}$ (G2), $1.12 \mu \mathrm{m}\left(\mathrm{G}_{3}\right)$ e 1.14 $\mu \mathrm{m}\left(\mathrm{G}_{4}\right)$. Porém, estes grupos não apresentaram diferenças estatisticamente significante entre si ( $p>0.05$ ).

Tabela 1 - Rugosidade superficial média $(\mathrm{Ra})$, em $\cdot \mathrm{m}$, e desvio-padrão $(\mathrm{dp})$ dos grupos experimentais, após os procedimentos de acabamento e polimento

\begin{tabular}{ccc}
\hline GRUPOS & MÉDIA (Ra) & DESVIO PADRÃO (dp) \\
\hline G1 (Controle) & $0.68^{\mathrm{a}}$ & 0.09 \\
G2 & $1.11^{\mathrm{b}}$ & 0.10 \\
G3 & $1.12^{\mathrm{b}}$ & 0.07 \\
G4 & $1.14^{\mathrm{b}}$ & 0.11 \\
\hline Médias seguidas de letras iguais indicam a ausência de diferença estatística significante (Teste de Tukey p>o.05).
\end{tabular}

\section{DISCUSSÃO}

Após a confecção da restauração em cerâmica e durante os procedimentos clínicos de cimentação, o ajuste com brocas diamantadas é inevitável, ${ }^{(2,3,8,81)}$ já que se faz necessário a correção de interferências na oclusão, características no contorno das margens das restaurações, melhora na aparência estética ou para correção de possíveis imperfeições, alterando a superfície da porcelana glazeada. ${ }^{(3,7,81,2)}$ Após a realização dos desgastes com as pontas diamantadas, a superfície da porcelana torna-se rugosa e extremamente abrasiva, revelando-se incompatível para estar presente em meio oral. ${ }^{(2,6,9,12)}$

Como relatado em alguns estudos, ${ }^{(5,6,8,8)}$ os desgastes realizados na superfície das cerâmicas exigem acabamento e polimento para proporcionar uma biocompatibilidade destas com os tecidos moles adjacentes. $\mathrm{O}$ estudo realizado por Vieira et al. ${ }^{(5)}$ comprova a necessidade de se utilizar polidores intraorais para promover lisura superficial na cerâmica, pois este mostrou altos valores de rugosidade superficial média $(\mathrm{Ra})$ nos grupos que não receberam polimento.
Por muito tempo houve uma preferência em realizar o acabamento final da cerâmica com um novo processo de glazeamento, fornecendo maior brilho às restaurações. Entretanto, mesmo o brilho sendo uma característica importante na estética para a reflexão da luz, muitas vezes o glaze modifica o valor das restaurações, o que acarreta em um reflexo maior que nos dentes naturais, transparecendo um aspecto artificial. $(2,7,8,10)$

De acordo com Karaksi et al,(7) Oliveira et al, (9) e Vieira et al, (5) o ideal é manter a superfície glazeada intacta, pois o glaze produz superfícies mais lisas, regulares e biocompatíveis que os processos de acabamento e polimento realizados. No presente estudo, o processo de glazeamento foi superior quando comparado com os demais sistemas de acabamento e polimento utilizados, pois apresentou menores valores de rugosidade superficial (Ra), o que também foi encontrado no estudo de Al-Wahadni. (4) Em contrapartida, Sarac et al.(11) afirmam, que as cerâmicas glazeadas não apresentaram diferença estatística significativa nos valores de rugosidade superficial quando comparadas com as cerâmicas que receberam diferentes tipos 
de acabamento e polimento. Já o estudo realizado por Netto Júnior et al. ${ }^{(13)}$ revela valores menores de rugosidade superficial para as cerâmicas polidas quando comparadas com as glazeadas.

Quando o processo de glazeamento foi questionado e deixou de ser preconizado no acabamento final das cerâmicas por uma economia de tempo clínico com os pacientes, ${ }^{(10)}$ surgiu a ideia de se utilizar pedras abrasivas, discos de óxido de alumínio e borrachas siliconizadas para polir as restaurações após o ajuste com as brocas diamantadas. ${ }^{(6,8)}$

Apesar de não existir um consenso entre os estudos publicados acerca da eficácia dos diferentes métodos de acabamento e polimento, ${ }^{(5,8,13-15)}$ encontra-se na literatura muitas opções de sistemas de polimento intraoral. Para a escolha destes, deve-se levar em conta o tipo de partícula abrasiva presente em sua composição e o método de utilização. ${ }^{(5)}$ Neste estudo, foram utilizados métodos com quatro passos para o polimento, bem como, partículas abrasivas pequenas que proporcionam um melhor polimento final. Os sistemas escolhidos são facilmente encontrados no mercado e de simples manuseio. $(5,7,12,13,16)$

Dentre os métodos encontrados na literatura, os discos de óxido de alumínio Sof-lex, segundo Gomis et al, ${ }^{(12)}$ apresentaram menores valores médios de rugosidade superficial, o que está de acordo com o presente estudo, no qual foi comprovado que ele não possui diferença estatisticamente significante em relação aos outros dois métodos utilizados. Entretanto, no estudo realizado por Oliveira et al(3) observou-se que os discos de alumínio Sof-lex foram os que apresentaram maior rugosidade, irregularidades e ranhuras na superfície das cerâmicas quando comparados com as borrachas abrasivas do sistema Shofu e Edenta.

Segundo Netto Júnior et al, ${ }^{(13)}$ as borrachas abrasivas da Edenta, tem sido um sistema de polimento de cerâmicas muito utilizado atualmente, estando presente não apenas neste estudo, mas também no de Oliveira et al, ${ }^{(9)}$ Oliveira et al, (3) e Vieira et al, (5) sendo eficaz, como comprovado neste estudo, na minimização da rugosidade superficial das cerâmi- cas, ainda que não consiga superar a lisura gerada pelo glaze. Dentre os três sistemas de acabamento e polimento utilizados neste estudo, o que apresentou o menor valor médio de rugosidade superficial foi o sistema de borrachas abrasivas Astropol, embora não tenha apresentado diferença estatisticamente significante com os demais sistemas. Os sistemas utilizados no presente trabalho foram escolhidos baseados na indicação clínica, relatos de utilização encontrados na literatura, baixo custo e quantidades similares de passos clínicos em sua aplicação.

A variedade dos resultados encontrados na literatura pode ser ocasionada pela utilização de diferentes produtos comerciais, metodologias e outras variáveis na manipulação e queima da cerâmica. $(5,13,17)$ Diante disto, se faz necessário a realização de mais estudos que proporcionem ao cirurgião-dentista maior precisão na escolha do sistema de polimento para cada tipo de cerâmica a ser utilizada.

\section{CONCLUSÃO}

Concluiu-se que os diferentes métodos de acabamento e polimento utilizados não foram capazes de promover uma superfície tão lisa quanto à aplicação do glaze, na cerâmica testada.

\section{REFERÊNCIAS}

1. Kelly JR, Benetti P. Ceramic materials in dentistry: historical evolution and current practice. Aust Dent J. 2011;56(1 Suppl):84-96.

2. Vieira $A C$, Oliveira MCS, Motta $C$ de AVB, Miranda CB, Lima EMCX. Eficácia dos sistemas de acabamento e polimento de cerâmicas odontológicas. Int J Dent. 2O11;1O(4):255-258.

3. Oliveira MCS, Santos GAG dos, Siqueira DV da S, Vieira AC, Oliveira VMB. Avaliação qualitativa da rugosidade superficial de uma porcelana odontológica após utilização de três diferentes sistemas de polimento. Odontol. Clín.-Cient. 2010;9(2):151-154. 
4. Al-Wahadni A. An in vitro investigation into the surface roughness of 2 glazed, unglazed, and refinished ceramic materials. Quintessence Int. 2006;37(5):311-317.

5. Vieira AC, Oliveira MSC, Lima EMCX, Rambob I, Leite M. Evaluation of the surface roughness in dental ceramics submitted to different finishing and polishing methods. J Indian Prosthodont Soc. 2013;13(3):290-295.

6. Camacho GB, Vinha D, Panzeri H, Nonaka T, Gonçalves M. Surface Roughness of a Dental Ceramic After Polishing with Different Vehicles and Diamond Pastes. Braz Dent J. 2006; 17(3): 191-194.

7. Karaksi $A O E$, Shehab GI, Eskander ME. Effect of reglazing and of polishing on the surface roughness of new ceramic restoration $(\mathrm{Hi}$ Ceran). Egypt Dent J. 1993;39 (3):485-490.

8. Benetti AR, Miranda CB, Ramos JRL. Avaliação da porosidade superficial da porcelana submetida a diferentes métodos de acabamento e polimento. PCL. Revista Brasileira de Prótese Clínica \& Laboratorial. 2002;22(4):489-493.

9. Oliveira MCS, Vieira AC, Miranda CB, Noya MS. The effect of polishing techniques on the surface roughness of a feldspathic porcelain. Rev. Odonto Ciênc. 2008; 23(4):330-332.

10. Ward MT, Tate WH, Power JM. Surface roughness of opalescent porcelains after polishing. Oper Dent. 1990;20(1):106-110.
11. Sarac D, Sarac YS, Yuzbasiogl E, Bal S. The effects of porcelain polishing systems on the color and surface texture of feldspathic porcelain. J Prosthet Dent. 2006;96(6):122-128.

12. Gomis JM, Bizar J, Anglada JM, Samso J, Peraire M. Comparative evaluation of four finishing systems on one ceramic surface. Int $\mathrm{J}$ Prosthodont. 2003;16(1):74-77.

13. Netto Júnior BA, Inove RT, Ribeiro FC, Feltrin PP. Estudo da rugosidade das superfícies metálica e porcelana mediante acabamento e polimento com sistemas de borrachas para polimento. UFES Rev. Odontol. 2006;8(1):6-18.

14. Patterson CJW, McLundie AC, Stirrups DR, Taylor WG. Efficacy of a porcelain refinishing system in restoring surface finish after grinding with fine and extra-fine diamond burs. J Prosthet Dent. 1992;68(6):4O2-4O6.

15. Chu FCS, Frankel N, Smales RJ. Surface roughness and flexural strength of self-glazed, polished, and reglazed In-Ceram/Vitadur Alpha porcelain laminates. Int J Prosthodont. 2000;13(2):66-71.

16. Wright MD, Masri R, Driscoll CF, Romberg E, Thompson CGA, Runyan CD. Comparison of three systems for the polishing of an ultralow fusing dental porcelain. J Prosthet Dent. 2004;92(4):486-490.

17. Jung M. Finishing and polishing of a hybrid composite and a heat-pressed glass ceramic. Oper Dent. 2002;27(3):175-183. 